\title{
On the Computational Security of a Distributed Key Distribution Scheme
}

\author{
Vanesa Daza, Javier Herranz, and Germán Sáez
}

\begin{abstract}
In a distributed key distribution scheme, a set of servers helps a set of users in a group to securely obtain a common key. Security means that an adversary who corrupts some servers and some users has no information about the key of a noncorrupted group. In this work, we formalize the security analysis of one such scheme [11] which was not considered in the original proposal. We prove the scheme is secure in the random oracle model, assuming that the Decisional Diffie-Hellman (DDH) problem is hard to solve. We also detail a possible modification of that scheme and the one in [24] which allows us to prove the security of the schemes without assuming that a specific hash function behaves as a random oracle. As usual, this improvement in the security of the schemes is at the cost of an efficiency loss.
\end{abstract}

Index Terms-Cryptography, key distribution, secret sharing schemes, provable security.

\section{INTRODUCTION}

$\mathrm{T}$ HERE are many situations where a group (or conference) of users needs a common secret key in order to use it for some purposes such as for secure communication by using symmetric encryption techniques or to allow the access to some restricted resource such as forums in the Internet or multiplayer computer games or, in general, to identify the members of the conference in front of each other or in front of external entities.

There are some different ways in which these users can securely obtain this common key, depending on the resources available to them. For example, in group key exchange protocols [7], the users broadcast partial values that allow all of them, after a certain number of rounds of communication, to securely compute the same group key. Current schemes [5], [22] work with only three or four rounds of communication.

Group key distribution schemes [12], [23] are also managed by the users of the group themselves, but are more suitable for dynamic situations, where users can join or leave the group at any period of time, which is decided by the own group members. This dynamism capability makes these schemes slightly different from group key exchange protocols, which must be reinitialized every time that the composition of the group changes.

- V. Daza is with the Departament d'Informàtica $i$ Matemàtiques, Universitat Rovira i Virgili (URV), Av. Paisos Catalans, 26, 43007, Tarragona, Spain.E-mail: vanesa.daza@urv.cat.

- J. Herranz is with the IIIA, Artificial Intelligence Research Institute, CSIC, Spanish National Research Council, Campus UAB s/n, E-08193 Bellaterra, Spain. E-mail: jherranz@iiia.csic.es.

- G. Sáez is with the Departament Matemàtica Aplicada IV, Universitat Politècnica de Catalunya (UPC), C/ Jordi Girona, 1-3 Campus Nord, Mòdul C-3, 08034 Barcelona, Spain. E-mail: german@ma4.upc.edu.

Manuscript received 29 Aug. 2005; revised 23 Nov. 2007; accepted 4 Feb. 2008; published online 20 Mar. 2008.

Recommended for acceptance by F. Lombardi.

For information on obtaining reprints of this article, please send e-mail to: tc@computer.org, and reference IEEECS Log Number TC-0288-0805.

Digital Object Identifier no. 10.1109/TC.2008.50.
A drawback of these two approaches is that there must be some kind of synchronization among the users because they must all execute the protocol at the same time. In some scenarios, this can be a problem and it is desirable that every user be able to obtain the conference key whenever he wants.

To achieve this property, Needham and Schroeder [25] proposed considering a key distribution center which computes and stores the conference keys and sends them to the users when they contact it. However, this solution has some drawbacks: The center is a bottleneck of the system, a possible point of failure, and a clear target for attacks because it knows all of the keys of the system.

To overcome these problems, Naor et al. [24] introduced the notion of distributed key distribution schemes. The task of the key distribution center is shared among a set of servers which independently send some information to the users, which allows them to compute the conference key. In a nutshell, the protocol is divided into three different phases. In the initialization phase, servers jointly generate some shared secret information. Later, in the key request phase, a user asks some servers for a conference key; servers check that this user actually belongs to the conference and, if so, each of them uses its share of the secret information to compute a partial conference key. In the key delivery phase, the user combines the received information to obtain the final conference key. A necessary property is that all of the users in the same conference must obtain the same key, independently of the set of servers that they contact.

\subsection{Related Work}

Distributed key distribution schemes have been considered in both information-theoretic [4], [10], [24] and computational [11], [13], [24] frameworks. The first proposal of distributed key distribution schemes in a computational framework was given in the original paper [24] as a natural application of the concept of threshold pseudorandom functions (TPRFs). Although the specific security of distributed key distribution schemes was not considered there, they proved that their TPRFs satisfy some properties 
(in the random oracle model and under the Decisional Diffie-Hellman (DDH) Assumption), which directly implies that the resulting distributed key distribution schemes are secure in the formal model defined some years later. The proposal in [24] pursues increasing server availability and decreasing network communication; it does not take very much consideration of the computational costs for servers and users.

In [11], an alternative scheme was proposed with the goal of reducing the computational load on the users, which was quite heavy in the proposals of [24]. The price for this improvement is increasing the computational and communication costs for the servers. The idea is that this different way of distributing the costs of the scheme can make more sense in practice because servers are assumed to be powerful devices, whereas users can be mobile devices (PDAs, mobile phones, etc.) with low computational resources. In such situations, a user can broadcast a query for a key to a set of servers and expects to receive the key quite directly, without needing to perform some extra operations to obtain it (other than decrypting). On the other hand, servers can be, in some way, paid for doing most of the work. However, the proposal in [11] did not include any formal security analysis.

The first explicit and formal security model for distributed key distribution schemes in the computational framework was given in [13]. Roughly speaking, a distributed key distribution scheme is secure if an adversary who corrupts some subset of servers and some conferences of users has no information about the key of a different, and noncorrupted, conference. In the same work, a general construction of secure distributed key distribution schemes starting from distributed signature schemes was provided.

\subsection{Our Contribution}

In this work, we formally prove that the scheme in [11] enjoys the security properties required for distributed key distribution schemes. The proof is by reduction to the hardness of the DDH problem in the random oracle model. We consider malicious adversaries and, so, we add mechanisms such as verifiable secret sharing and zeroknowledge proofs of knowledge to detect dishonest participants.

We also detail how to modify a part of the scheme in order to avoid the use of a specific hash function, which is modeled as a random oracle in the security proofs. This modification was already mentioned (briefly) in [24] and is valid for the scheme proposed there as well. As usually happens, the improvement in the security of the schemes is at the cost of a reduction in their efficiency because now the servers must maintain a table where each conference is assigned one value jointly chosen by them.

\subsection{Organization of This Paper}

In Section 2, we describe the framework of distributed key distribution schemes: the protocols that define such schemes and the formal security requirements that they must satisfy. Then, in Section 3, we recall ElGamal encryption and a protocol for the joint generation of a shared secret value because they are essential tools of the scheme that we study. In Section 4, we review the distributed key distribution scheme proposed in [11] and then we analyze it: We add the security analysis of the employed protocol to compute proofs of knowledge, which provides robustness to the scheme, and we prove that the whole scheme is secure in the random oracle model. We propose, in Section 5, a slight modification to the schemes in [11] and [24], which allows us to prove their security without the (sometimes too strong) assumption that a hash function behaves as a random oracle. Finally, we devote Section 6 to the conclusions of this work.

\section{Distributed Key Distribution Schemes}

Naor et al. introduced the notion of distributed key distribution schemes in [24]. In this kind of scheme, we have a set $\mathcal{U}=\left\{U_{1}, \ldots, U_{m}\right\}$ of $m$ users and a set $\mathcal{S}=$ $\left\{S_{1}, \ldots, S_{n}\right\}$ of $n$ servers. Users in the same conference $C \subset \mathcal{U}$ want to obtain a common secret key, $\kappa_{C}$, for example, in order to communicate securely with the other members of the conference or to use it as an access key for some restricted resources. To obtain $\kappa_{C}$, users must interact with some servers in $\mathcal{S}$. The subsets of servers that are allowed to provide conference keys are those in the access structure $\Gamma \subset 2^{\mathcal{S}}$, which must be monotone increasing: If $A_{1} \subset A_{2}$ and $A_{1} \in \Gamma$, then $A_{2} \in \Gamma$.

In more detail, a distributed key distribution scheme consists of the following phases:

- Initialization phase. This phase involves only the servers, which interact with each other. The input is the access structure $\Gamma$ and the output contains some public information and partial secret information for each server $S_{i} \in \mathcal{S}$.

- Key request phase. In this phase, a user $U_{j}$ contacts a subset of servers in order to obtain a conference key for some conference $C$ to which he belongs. Servers send to $U_{j}$ some value(s) which depend on their partial secret information, on the identity of $U_{j}$, and on the conference $C$.

- Key delivery phase. Finally, the user $U_{j}$ combines the received values and tries to compute the conference key $\kappa_{C}$ for the conference $C$. If the contacted subset of servers belongs to the access structure $\Gamma$, then $U_{j}$ is always able to compute the correct $\kappa_{C}$.

Communication Model. We assume the following communication model for the distributed key distribution scheme that we study in this paper. On the one hand, communication between servers is done through secret and authenticated channels, not necessarily synchronous. On the other hand, communication between servers and users requires authenticated channels, but does not require secret or synchronous channels.

\subsection{Security of Distributed Key Distribution Schemes}

The level of security required for distributed key distribution schemes depends on the scenario where the conference members use the generated key and basically on the type of attacks the application must be protected against. A high 
enough (and standard) level of security is the equivalent of semantic security for encryption schemes [20]. Roughly speaking, an adversary should not be able to distinguish a conference key $\kappa_{C}$ from a value taken at random from the set of possible keys, even if it corrupts some subset of servers and the protocol is executed for other conferences. The adversary can require the execution of the protocol for conferences of his choice, as many times as he wants; this corresponds to a dishonest user who starts many sessions of the same application, with different conferences.

\subsubsection{The Adversarial Model}

Obviously, the number of servers that an adversary can corrupt must be limited; otherwise, if he controls, for example, an authorized set of servers in $\Gamma$, he can compute all of the conference secret keys and there is no security at all. Therefore, an adversary structure $\mathcal{A} \subset 2^{\mathcal{S}}$ must be defined, containing those subsets of dishonest servers that the system is able to tolerate. It must be monotone decreasing: If a subset $B$ can be corrupted by $\mathcal{A}$, then any subset contained in $B$ can also be corrupted by $\mathcal{A}$. A trivial condition to ensure the security of the schemes in this scenario is that $\mathcal{A} \cap \Gamma=\emptyset$.

We deal with static but malicious adversaries. Static means that the set of corrupted servers is chosen at the beginning of the life of the system and remains fixed from that moment on. Malicious means that the adversary can put together the private information of the corrupted parties and can force them to deviate from the correct execution of the protocols. In this case, a standard requirement for the designed protocols is that of robustness. There must be mechanisms to detect the corrupted parties which do not follow the protocol correctly; once this is done, there must remain enough honest parties to successfully finish the execution of the protocol. A necessary condition to ensure robustness is $\mathcal{A}^{c} \subset \Gamma$, where $\mathcal{A}^{c}=\{\mathcal{P}-B \mid B \in \mathcal{A}\}$. Throughout this paper, since we want robustness, we will assume that this condition is satisfied by the structures considered in the protocols.

\subsubsection{Formal Security Model for Distributed Key Distribution Schemes}

The adversary against the distributed key distribution scheme will be denoted as $\mathcal{F}$. Such an adversary $\mathcal{F}$ is given the public parameters of the system: the set of servers $\mathcal{S}=\left\{S_{1}, \ldots, S_{n}\right\}$, the access and adversary structures $\Gamma \subset$ $2^{\mathcal{S}}$ and $\mathcal{A} \subset 2^{\mathcal{S}}$, and the set of users $\mathcal{U}=\left\{U_{1}, \ldots, U_{m}\right\}$.

Extending the security model introduced in [13], inspired by other works on key distribution (see [1], for example), we describe here a game $\mathcal{G}$, played by an adversary $\mathcal{F}$ against a distributed key distribution scheme. We consider schemes which fall inside the model explained in this work, with an initialization phase performed only by servers, a key request and computational phase where servers and users interact, and a key delivery phase where users compute the conference keys. The game $\mathcal{G}$, which captures the capabilities (requesting the execution of the protocol, Step 3) and the goals (distinguishing a conference key from a random key, Steps 4-6) of the adversary $\mathcal{F}$, works as follows:
1. The adversary chooses a subset of servers $B \in \mathcal{A}$ to be corrupted.

2. The initialization phase of the distributed key distribution scheme, which involves only servers, is executed. The adversary $\mathcal{F}$ obtains all of the public information and the secret information of the corrupted servers $S_{j} \in B$.

3. The adversary $\mathcal{F}$ adaptively chooses $Q_{\text {conf }}$ different conferences $C^{\prime} \subset \mathcal{U}$. The key request and the computational phase of the scheme, as well as the key delivery phase, are executed for each user of these conferences, as many times as $\mathcal{F}$ wants. As a result, $\mathcal{F}$ obtains all of the information produced in these executions. One could think that the adversary has corrupted all members of such conferences $C^{\prime} . \mathcal{F}$ obtains the private information generated by the corrupted servers, the information made public by all of the servers, the information obtained by users in $C^{\prime}$ (including the resulting conference key $\kappa_{C^{\prime}}$ ), etc.

4. The adversary chooses a conference $C$ different from the conferences it has queried in the previous step. For this conference, the key request and computational phase of the scheme is executed. The adversary $\mathcal{F}$ obtains the private information of the corrupted servers and the information broadcast by all the servers, but it has no access to the secret information received by users in $C$. This Step 4 can be executed in parallel to Step 3 to allow concurrent attacks. Furthermore, more key conference queries can be made by the adversary after this step, for conferences $C^{\prime} \neq C$, which are answered as in Step 3.

5. A random bit $b^{*} \in\{0,1\}$ is chosen by a challenger. If $b^{*}=1$, the adversary $\mathcal{F}$ is given the real key $\kappa_{C}$. If $b^{*}=0$, the adversary is given an element taken uniformly at random from the set of possible conference keys.

6. The adversary $\mathcal{F}$ outputs a bit $b^{\prime} \in\{0,1\}$, with $b^{\prime}=1$ if $\mathcal{F}$ thinks that the received element is actually $\kappa_{C}$ and $b^{\prime}=0$ otherwise. It wins the game if $b^{\prime}=b^{*}$.

We define the advantage of such an adversary $\mathcal{F}$ in breaking the semantic security of the distributed key distribution scheme as

$$
\varepsilon=\operatorname{Pr}\left[b^{\prime}=b^{*}\right]-\frac{1}{2} .
$$

Definition 1. An adversary $\mathcal{F}\left(\mathcal{U}, \mathcal{S}, \Gamma, \mathcal{A}, T, \varepsilon, Q_{\text {conf }}\right)$-breaks the distributed key distribution scheme if it plays game $\mathcal{G}$ in time at most $T$ and its advantage in breaking the semantic security of the scheme is at least $\varepsilon$.

Regarding the protocol of Naor et al. [24], they actually propose TPRFs which, in particular, can be easily transformed into distributed key distribution schemes. They define the security requirements for such functions and prove that their proposal of TPRF satisfies these requirements, in the random oracle model, under the DDH Assumption. It is easy to see that this fact directly implies that the resulting distributed key distribution scheme is secure in this formal model. 
For simplicity, we have decided to concentrate on the case of one single session for each conference. In the case of the studied scheme, one possibility to deal with different sessions $s$ would be to include another input to the hash function $H$, that is, the conference key for $C$ in the session $s$ would be $\kappa_{C, s}=H(C, s)^{\alpha}$. Since, in the security proof, we will assume that $H$ behaves as a random oracle, this solution would be secure. The same technique could be applied to the scheme in [24], in order to allow different sessions.

\section{BUILDING BLOCKS}

In this section, we review two cryptographic primitives that will appear in the explanation of the proposal of the distributed key distribution scheme that we study in this work.

\subsection{EIGamal Encryption}

In [17], ElGamal proposed a public-key probabilistic encryption scheme. The public parameters of the scheme are two large primes, $p$ and $q$, such that $q \mid p-1$, and an element $g$ verifying that the multiplicative subgroup $\mathbb{G}=$ $\langle g\rangle$ of $\mathbb{Z}_{p}^{*}$ has order $q$ (this mathematical framework will be common to all of the protocols explained in this work).

Every user $U$ generates both his public and private keys by choosing a random element $x \in \mathbb{Z}_{q}^{*}$ and computing $y=g^{x} \bmod p$. The public key of user $U$ is $y$ and his private key is $x$.

If someone wants to encrypt a message $m \in \mathbb{G}$ for user $U$, he chooses a random element $\beta \in \mathbb{Z}_{q}^{*}$ and computes $r=$ $g^{\beta} \bmod p$ and $s=m y^{\beta} \bmod p$. The ciphertext of message $m$ that is sent to user $U$ is $c=(r, s)$.

When $U$ wants to recover the original message $m$ from the ciphertext $c=(r, s)$, he computes

$$
m=s r^{-x} \bmod p .
$$

The semantic security of the ElGamal cryptosystem is equivalent to the DDH Assumption [14], which states that the DDH problem is hard to solve.

Definition 2 (DDH problem). We say that an algorithm is an $(\varepsilon, T)$-solver of the DDH problem if it runs in time at most $T$ and distinguishes with probability at least $1 / 2+\varepsilon$ between the two probability distributions $\mathcal{D}_{D H}$ and $\mathcal{D}_{\text {rand }}$, where $\mathcal{D}_{D H}=\left(g^{a}, g^{b}, g^{a b}\right)$, for uniformly and independently chosen values $a, b \in \mathbb{Z}_{q}$, and $\mathcal{D}_{\text {rand }}=\left(g^{a}, g^{b}, g^{c}\right)$, for uniformly and independently chosen values $a, b, c \in \mathbb{Z}_{q}$.

\subsection{Secret Sharing Techniques}

In this section, we explain some basics on secret sharing schemes. These schemes can also be used by a set of players to jointly generate shares of a random secret value $\alpha \in \mathbb{Z}_{q}$ such that the value $y=g^{\alpha} \bmod p$ is public.

In a secret sharing scheme, a dealer (usually denoted by $D$ ) distributes shares of a secret value among a set of players $\mathcal{P}=\left\{P_{1}, \ldots, P_{n}\right\}$ in such a way that only authorized subsets of players (those in the monotone increasing access structure $\Gamma \subset 2^{\mathcal{P}}$ ) can recover the secret value from their shares, whereas nonauthorized subsets do not obtain any information about the secret.
Secret sharing schemes were introduced independently by Shamir [29] and Blakley [3] in 1979. Shamir considered threshold access structures $\Gamma=\{A \subset \mathcal{P}:|A| \geq t+1\}$, defined by a threshold $t+1$, and proposed a secret sharing scheme for these structures based on polynomial interpolation. Other works have proposed schemes realizing more general access structures, such as vector space secret sharing schemes [6]. An access structure $\Gamma$ is realizable by such a scheme, in a finite field $\mathbb{Z}_{q}$ for some prime $q$, if there exists a positive integer $r$ and an assignment of vectors $\psi$ : $\mathcal{P} \cup\{D\} \longrightarrow\left(\mathbb{Z}_{q}\right)^{r}$ (one for each participant) such that $A \in$ $\Gamma$ if and only if $\psi(D)$ is a linear combination of the vectors in $\left\{\psi\left(P_{i}\right)\right\}_{P_{i} \in A}$. Here, $D$ denotes a special entity (real or not), outside the set $\mathcal{P}$. If a real dealer $D$ wants to share a secret $k \in \mathbb{Z}_{q}$ among the players in $\mathcal{P}$, he chooses a random vector $\mathbf{v} \in\left(\mathbb{Z}_{q}\right)^{r}$ such that $\mathbf{v} \cdot \psi(D)=k$. The share of each player $P_{i} \in \mathcal{P}$ is $k_{i}=\mathbf{v} \cdot \psi\left(P_{i}\right)$. If $A \in \Gamma$, there exist values $\left\{\lambda_{i}^{A}\right\}_{P_{i} \in A}$ such that $\psi(D)=\sum_{P_{i} \in A} \lambda_{i}^{A} \psi\left(P_{i}\right)$. Then, it is easy to see that $k=\sum_{P_{i} \in A} \lambda_{i}^{A} k_{i} \bmod q$. Using simple linear algebra, one can also see that subsets out of $\Gamma$ obtain no information at all about the secret value $k$.

Simmons et al. [30] introduced linear secret sharing schemes, which can be seen as a generalization of vector space secret sharing schemes where each player can be assigned more than one vector (and, therefore, the length of each share can be larger than the secret). They proved that any access structure can be realized by a linear secret sharing scheme, but the scheme is, in general, quite inefficient in the sense that the rate between the length of the secret and the length of the shares is very low.

From now on in our work, we will consider any possible access structure $\Gamma$, so we will know that there exists a linear secret sharing scheme realizing this structure. For simplicity, we will suppose that this scheme is a vector space scheme defined by a function $\psi$. All of the results are valid in the more general case where each player is associated with more than one vector as well.

\subsubsection{Joint Generation of a Random Shared Secret Value}

If the secret value to be shared is a random number from the corresponding finite field, the generation and distribution of shares in a secret sharing scheme can be performed by the players themselves, without any external party (like the dealer).

Now, we explain the protocol for the joint generation of a random secret value $\alpha$, shared among players in $\mathcal{P}$ according to the access structure $\Gamma$. The protocol is secure against the action of a malicious adversary who can corrupt a subset of players in the adversary structure $\mathcal{A}$. An obvious required condition to ensure the privacy of the final secret is $\Gamma \cap \mathcal{A}=\emptyset$. We assume that $\Gamma$ can be realized by a vector space secret sharing scheme defined by a mapping $\psi: \mathcal{P} \cup\{D\} \rightarrow\left(\mathbb{Z}_{q}\right)^{r}$. Some other parameters are public, like $p, q$, and two elements $g$, $h$ such that $\mathbb{G}=\langle g\rangle$ has order $q$ and $h \in \mathbb{G}$. The protocol, which is a generalization of the threshold protocol in [19], consists of the following steps:

1. Each player $P_{i} \in \mathcal{P}$ chooses at random a value $k_{i} \in$ $\mathbb{Z}_{q}$ and distributes it among all players in $\mathcal{P}$, with the 
following verifiable secret sharing technique (which is a generalization of [26]):

a. $P_{i}$ chooses two random vectors $\mathbf{v}_{\mathbf{i}}=$ $\left(v_{i}^{(1)}, \ldots, v_{i}^{(r)}\right)$ and $\quad \mathbf{w}_{\mathbf{i}}=\left(w_{i}^{(1)}, \ldots, w_{i}^{(r)}\right) \quad$ in $\left(\mathbb{Z}_{q}\right)^{r}$ such that $\mathbf{v}_{\mathbf{i}} \cdot \psi(D)=k_{i}$. Then, $P_{i}$ sends to each player $P_{j}$ in $\mathcal{P}$ the pair $\left(k_{i j}, k_{i j}^{\prime}\right)=\left(\mathbf{v}_{\mathbf{i}} \cdot \psi(j), \mathbf{w}_{\mathbf{i}} \cdot \psi(j)\right)$. He also makes public the commitments $V_{i \ell}=g^{v_{i}^{(\ell)}} h^{w_{i}^{(\ell)}}$, for $1 \leq \ell \leq r$.

b. Each player $P_{j} \in \mathcal{P}$ verifies the correctness of his share $k_{i j}$ by checking that

$$
g^{k_{i j}} h^{k_{i j}^{\prime}}=\prod_{\ell=1}^{r}\left(V_{i \ell}\right)^{\psi\left(P_{j}\right)^{(\ell)}},
$$

where $\psi\left(P_{j}\right)^{(\ell)}$ is the $\ell$ th component of the vector $\psi\left(P_{j}\right)$. If this check fails, $P_{j}$ makes public a complaint against $P_{i}$.

c. For any complaint that $P_{i}$ receives from some player $P_{j}$, he must broadcast a pair of values $\left(k_{i j}, k_{i j}^{\prime}\right)$ satisfying (1).

d. Player $P_{i}$ is marked as disqualified if he receives a complaint from a set of players out of $\mathcal{A}$ or if he answers some complaint with values which do not satisfy (1).

2. Each player defines the set of nondisqualified players Qual. Since $\mathcal{A}^{c} \subset \Gamma$, we have that Qual $\in \Gamma$. Furthermore, for all players $P_{i} \in$ Qual that pass this phase, there are valid shares $k_{i j}$ corresponding to players $P_{j}$ that form an authorized subset.

3. The generated random secret will be $\alpha=\sum_{P_{i} \in Q u a l} k_{i}$. Each player $P_{j} \in \mathcal{P}$ computes his share $\alpha_{j}$ of the secret $\alpha$ as $\alpha_{j}=\sum_{P_{i} \in Q u a l} k_{i j}$.

4. Now, the players want to compute the value $y=g^{\alpha}=\prod_{P_{i} \in Q u a l} g^{k_{i}} \in \mathbb{Z}_{p}^{*}$. They use Feldman's verifiable secret sharing scheme (see [15] for the original threshold version):

a. Each player $P_{i} \in Q u a l$ broadcasts $A_{i \ell}=g^{v_{i}^{(\ell)}}$, for $1 \leq \ell \leq r$.

b. Each player $P_{j} \in$ Qual verifies if

$$
g^{k_{i j}}=\prod_{\ell=1}^{r}\left(A_{i \ell}\right)^{\psi\left(P_{j}\right)^{(\ell)}} .
$$

If this check fails, player $P_{j}$ complains by broadcasting the pair $\left(k_{i j}, k_{i j}^{\prime}\right)$ that satisfies (1) but does not satisfy (2).

c. If player $P_{i}$ receives some valid complaint at Step 4.b, the other players $P_{j} \in$ Qual run the reconstruction phase of Pedersen's scheme to recover $k_{i}$ and, in particular, $A_{i}=g^{k_{i}}$. Otherwise, the value $g^{k_{i}}$ is computed from the commitments $A_{i \ell}$ and the public vector $\psi(D)$ as

$$
g^{k_{i}}=g^{\mathbf{v}_{\mathbf{i}} \cdot \psi(D)}=\prod_{\ell=1}^{r} g^{v_{i}^{(\ell)} \psi(D)^{(\ell)}}=\prod_{m=1}^{t}\left(A_{i \ell}\right)^{\psi(D)^{(\ell)}} .
$$

d. Finally, the value $y=g^{\alpha}$ can be computed as

$$
y=g^{\sum_{P_{i} \in \text { Qual }} k_{i}}=\prod_{P_{i} \in \text { Qual }} g^{k_{i}} .
$$

Something similar happens with the values

$$
D_{j}=g^{\alpha_{j}}=g^{\sum_{P_{i} \in Q u a l} k_{i j}},
$$

which can be publicly computed from the values $g^{k_{i j}}$ obtained in Steps 4.b and 4.c. We denote the output of this protocol with the expression:

$$
\left(\alpha_{1}, \ldots, \alpha_{n}\right) \stackrel{(\mathcal{P}, \Gamma, \mathcal{A})}{\longrightarrow}\left(\alpha, g^{\alpha},\left\{D_{j}\right\}_{1 \leq j \leq n}\right),
$$

where $\alpha_{i}$ is the share of the secret $\alpha$ held by player $P_{i}$ and the values $g^{\alpha}$ and $\left\{D_{j}\right\}_{1 \leq j \leq n}$ are publicly known.

It is not difficult to see that this protocol is simulatable in the following sense: Given a corruptible subset $B \in \mathcal{A}$ and a value $Y \in \mathbb{G}$, it is possible to simulate, in polynomial time, values which are indistinguishable from the ones that an adversary corrupting players in $B$ would obtain from an execution of the protocol which outputs $g^{\alpha}=Y$. We denote all this simulated information by $\operatorname{Sim}(B, Y)$. This fact ensures that the protocol is secure because an adversary does not obtain any information from the execution of the protocol that he could not produce by himself.

The proof of this fact is omitted here by lack of originality since it is basically a rewriting of the proof of simulatability of the protocol in [19], where threshold secret sharing is replaced with linear secret sharing. Note that this change does not add any new subtle issue to the proof in [19], which had been proposed to correct some flaws in previous wellknown protocols for distributed key generation [18], [26].

\section{The Distributed Key Distribution Scheme OF DAZA ET AL.}

In this section, we analyze the scheme proposed in [11]. The goal of this paper is to prove the security of this scheme because [11] does not include any security analysis. After reviewing and modifying the protocols of the scheme, we first prove that the employed proof of knowledge is secure. Then, in Section 4.3, we will prove that the whole distributed key distribution scheme is secure.

In the original proposal of Naor et al. [24], servers must send the partial information to users throughout private channels. In practice, this can be done, for example, by using a suitable encryption scheme. Then, the user must check the correctness of the received values and combine some correct values to obtain the final key; this step involves some modular exponentiations, which can be costly to the user if he does not have enough computational resources.

The goal of the scheme by Daza et al. [11] is to reduce the computational effort required to the users. The idea is as follows: Since the information received by users must be encrypted anyway, an encryption scheme such as ElGamal cryptosystem can be used. In general, any multiplicatively homomorphic encryption scheme $E_{P K}(m)$ satisfying 
$E_{P K}\left(m_{1} \cdot m_{2}\right)=E_{P K}\left(m_{1}\right) \cdot E_{P K}\left(m_{2}\right)$ could be used. The partial encryptions computed by the servers can be combined by the servers themselves (with one extra round of communication) in order to produce an encryption of the final conference key. This value is sent to the user, who is the only one who can decrypt it and obtain the key. In this way, the number of operations that users must perform is significantly reduced at the cost of increasing the computational and communication effort required to servers. Note that this may make sense in real scenarios where users have low computational resources and servers are paid in some way for doing this work.

\subsection{The Scheme}

Let $\mathcal{U}=\left\{U_{1}, \ldots, U_{m}\right\}$ and $\mathcal{S}=\left\{S_{1}, \ldots, S_{n}\right\}$ be the sets of users and servers. Let $\Gamma, \mathcal{A} \subset 2^{\mathcal{S}}$ be the access and adversary structures satisfying $\mathcal{A} \cap \Gamma=\emptyset$ and $\mathcal{A}^{c} \subset \Gamma$. Again, we assume that the access structure $\Gamma$ can be realized by a vector space secret sharing scheme. That is, there exist a positive integer $r$ and a function $\psi$ : $\mathcal{S} \cup\{D\} \longrightarrow\left(\mathbb{Z}_{q}\right)^{r}$ such that $A \in \Gamma$ if and only if $\psi(D) \in\left\langle\psi\left(S_{i}\right)\right\rangle_{S_{i} \in A}$.

We denote by $\Omega=\Omega(\Gamma, \mathcal{A})=\{R \subset \mathcal{P} \mid R-B \in \Gamma$, for all $B \in \mathcal{A}\} \subset \Gamma$ the monotone increasing family of robust subsets. Since $\Gamma$ and $\mathcal{A}$ satisfy $\mathcal{A}^{c} \subset \Gamma$, we know that $\mathcal{P} \in \Omega$ and so the family $\Omega$ is not empty. In the scheme, a user who wants to know a conference key must contact with the servers of some robust subset $R \in \Omega$.

Let $H$ be a hash function (collision and preimage resistant) that inputs a conference $C \subset \mathcal{U}$ and outputs an element in $\mathbb{G}=\langle g\rangle \subset \mathbb{Z}_{p}^{*}$. In the security proof of the scheme, this hash function is assumed to behave as a random oracle (following a usual methodology introduced in [2]).

Each user $U \in \mathcal{U}$ has an ElGamal secret key $x \in \mathbb{Z}_{q}^{*}$ and the matching public key is $y=g^{x}$. The parameters $(p, q, \mathbb{G}=\langle g\rangle)$ are the same for all of the servers and users in the system.

Initialization phase. In order to compute their secret shares, servers in $\mathcal{S}$ jointly execute the protocol

$$
\left(\alpha_{1}, \ldots, \alpha_{n}\right) \stackrel{(\mathcal{S}, \Gamma, \mathcal{A})}{\longleftrightarrow}\left(\alpha, g^{\alpha},\left\{D_{j}\right\}_{1 \leq j \leq n}\right),
$$

where $\alpha, \alpha_{i} \in \mathbb{Z}_{q}$ are random, as explained in Section 3.2.1.

Note that, in the proposal published in [11], a different (and simpler) protocol for the joint generation of a random secret is used. However, when analyzing security, one realizes that the simpler protocol is not simulatable and, therefore, the security of the distributed key distribution scheme as it is in [11] cannot be proved. However, the protocol explained in Section 3.2.1 is simulatable and the whole scheme can be proven secure, as we will see in Section 4.3.

Key request and computational phase. A user $U$ in a conference $C \subset \mathcal{U}$ asks for the conference key $\kappa_{C}$ to a robust subset of servers $R \in \Omega$ :

1. Each server $S_{i} \in R$ applies the hash function $H$ to the conference $C$, obtaining $h_{C}=H(C) \in \mathbb{G}$. The conference key will be $\kappa_{C}=h_{C}^{\alpha}$.

2. Then, server $S_{i} \in R$ encrypts the value $h_{C}^{\alpha_{i}}$ using the ElGamal public key $y$ of user $U$. That is,
- $\quad$ server $S_{i}$ chooses a random element $\beta_{i} \in \mathbb{Z}_{q^{\prime}}^{*}$

- $\quad$ it computes $r_{i}=g^{\beta_{i}}$ and $s_{i}=h_{C}^{\alpha_{i}} y^{\beta_{i}}$, and

- $\quad$ server $S_{i}$ broadcasts the ciphertext $c_{i}=\left(r_{i}, s_{i}\right)$.

3. Server $S_{i}$ computes a noninteractive and zeroknowledge proof of the knowledge of values $\alpha_{i}$ and $\beta_{i}$, which make the ciphertext $c_{i}$ consistent:

$$
\begin{aligned}
\operatorname{Proof}_{i} & =P K\left\{\left(\alpha_{i}, \beta_{i}\right): D_{i}=g^{\alpha_{i}} \wedge r_{i}=g^{\beta_{i}} \wedge s_{i}\right. \\
& \left.=h_{C}^{\alpha_{i}} y^{\beta_{i}}\right\} .
\end{aligned}
$$

More details about the design and security of this proof of knowledge, which makes use of a hash function $\hat{H}:\{0,1\}^{*} \rightarrow \mathbb{Z}_{q}$, are given in Section 4.2.

4. Each server $S_{i} \in R$ verifies the proofs published by the rest of the servers until it obtains correct partial ciphertexts from an authorized subset $A \in \Gamma$. Notice that such a subset in $\Gamma$ always exists because $R \in \Omega$ and, therefore, $R-B \in \Gamma$ for any possible subset $B$ of incorrect partial ciphertexts computed by corrupted servers.

Since $A \in \Gamma$, we know that there exist values $\left\{\lambda_{j}^{A}\right\}_{S_{j} \in A}$ such that $\alpha=\sum_{S_{j} \in A} \lambda_{j}^{A} \alpha_{j}$.

5. Using homomorphic properties of ElGamal encryption, server $S_{i}$ can compute an encryption $(r, s)$ of the conference key $\kappa_{C}=h_{C}^{\alpha}$ from the values $c_{j}$ broadcast by servers $S_{j} \in A$ as follows:

$$
\begin{aligned}
& r=\prod_{S_{j} \in A} r_{j}^{\lambda_{j}^{A}}=g^{\sum_{S_{j} \in A} \lambda_{j}^{A} \beta_{j}}, \\
& s=\prod_{S_{j} \in A} s_{j}^{\lambda_{j}^{A}}=h_{C}^{\sum_{S_{j} \in A} \lambda_{j}^{A} \alpha_{j}} y^{\sum_{S_{j} \in A} \lambda_{j}^{A} \beta_{j}}=h_{C}^{\alpha} y^{\sum_{S_{j} \in A} \lambda_{j}^{A} \beta_{j}} .
\end{aligned}
$$

Since the elements $\left\{\beta_{j}\right\}_{S_{j} \in A}$ are random, the element $\sum_{S_{j} \in A} \lambda_{j}^{A} \beta_{j}$ is also random and, so, $(r, s)$ is a valid ElGamal encryption of the message $h_{C}^{\alpha}$. We also note that the resulting ciphertext $(r, s)$ does not depend on the considered authorized subset $A \in \Gamma$.

Key delivery phase. Each server in $R$ sends to user $U$ the ciphertext $c=(r, s)$ that he has computed. User $U$ selects a value that has been sent by all of the servers of some subset out of $\mathcal{A}$ (this means that there exists at least one honest server in this subset and, so, the corresponding ciphertext must be the correct one). The user decrypts it and obtains the conference key $\kappa_{C}=h_{C}^{\alpha}$. Note that, as desired, the obtained key does not depend on the set of servers who have participated in the execution of the protocol.

\subsection{The Proof of Knowledge}

In the protocol described above, each server $S_{i}$ must compute the proof

$$
\operatorname{Proof}_{i}=\operatorname{PK}\left\{\left(\alpha_{i}, \beta_{i}\right): D_{i}=g^{\alpha_{i}} \wedge r_{i}=g^{\beta_{i}} \wedge s_{i}=h_{C}^{\alpha_{i}} y^{\beta_{i}}\right\} .
$$

This proof can be computed using a standard strategy [8], [16], [28] that transforms an interactive proof of knowledge 
into a noninteractive one by using a hash function $\hat{H}$, which is modeled in the security analysis as a random oracle.

For some known values $A, B, C, g_{1}, g_{2}, g_{3} \in \mathbb{G}$, the proof

$$
P K\left\{(\alpha, \beta): A=g_{1}^{\alpha} \wedge B=g_{1}^{\beta} \wedge C=g_{2}^{\alpha} g_{3}^{\beta}\right\}
$$

can be computed as follows: Let $\hat{H}:\{0,1\}^{*} \rightarrow \mathbb{Z}_{q}$ be a hash function. The prover does the following:

1. Choose uniformly and at random $u, v \in \mathbb{Z}_{q}^{*}$.

2. Compute $T_{1}=g_{1}^{u}, T_{2}=g_{1}^{v}$, and $T_{3}=g_{2}^{u} g_{3}^{v}$.

3. Compute $h=\hat{H}\left(A, B, C, g_{1}, g_{2}, g_{3}, T_{1}, T_{2}, T_{3}\right)$.

4. Compute $w_{1}=u-\alpha h \bmod q$ and $w_{2}=v-\beta h \bmod q$.

5. The proof of knowledge is the tuple $\left(h, w_{1}, w_{2}\right)$.

The verifier of the proof must check if

$$
h=\hat{H}\left(A, B, C, g_{1}, g_{2}, g_{3}, g_{1}^{w_{1}} A^{h}, g_{1}^{w_{2}} B^{h}, g_{2}^{w_{1}} g_{3}^{w_{2}} C^{h}\right) .
$$

If so, then he accepts the proof; otherwise, he rejects the proof.

A zero-knowledge proof of knowledge must satisfy three properties: correctness, which means that the verifier always accepts the proof if the prover knows the secret values; soundness, which means that, if a prover does not know the secrets, then the probability that he computes a proof that makes the verifier accept is negligible; and, finally, zero-knowledge means that the only information that the verifier obtains from the execution of the protocol is if the prover knows the stated secret values or not.

The correctness of the protocol explained above is trivially fulfilled. We next prove that the protocol also achieves the other two properties in the random oracle model [2], where a hash function is seen as a totally random function.

Soundness. We prove that an algorithm $\mathcal{B}$ which has probability $\varepsilon$ to compute a valid proof knows the values $\alpha$ and $\beta$ with probability $\varepsilon^{2} / 5$.

In effect, the randomness of algorithm $\mathcal{B}$ consists of the randomness of the values output by the (random) hash function $\hat{H}$ and the own randomness of $\mathcal{B}$. If an execution of $\mathcal{B}$ produces a tuple $\left(h, w_{1}, w_{2}\right)$, then we denote as $X$ the randomness employed by $\mathcal{B}$ just before asking to the random oracle the value $\hat{H}\left(A, B, C, g_{1}, g_{2}, g_{3}, g_{1}^{w_{1}} A^{h}, g_{1}^{w_{2}} B^{h}, g_{2}^{w_{1}} g_{3}^{w_{2}} C^{h}\right)$. We denote as $Y$ the rest of randomness employed by $\mathcal{B}$ (including the value output by the random oracle in the aforementioned query).

We denote as $W \subset X \times Y$ the set of random choices (or executions of $\mathcal{B}$ ) that lead to a valid proof of knowledge. By hypothesis, we have that $\operatorname{Pr}[W]=\varepsilon$. We apply to this situation the well-known splitting lemma (see, for example, [27]), also known as heavy-row lemma.

Then, there exists $V \subset W$ such that, for all $(x, y) \in V$, we have

$$
\underset{y^{\prime}}{\operatorname{Pr}}\left[\left(x, y^{\prime}\right) \in W\right] \geq \frac{\varepsilon}{2} \quad \text { and } \quad \operatorname{Pr}[V \mid W] \geq \frac{1}{2} .
$$

When we execute $\mathcal{B}$ with randomness $(x, y)$, we will obtain $(x, y) \in V$ with probability at least $\varepsilon / 2$. In this case, we execute $\mathcal{B}$ again with fixed $x$ and random $y^{\prime}$. With probability at least $\varepsilon / 2$, the new execution $\left(x, y^{\prime}\right)$ belongs to $W$, that is, it also leads to a valid proof of knowledge.
Since $\hat{H}$ and $\hat{H}^{\prime}$ are random functions, the probability that

$$
\begin{aligned}
h & =\hat{H}\left(A, B, C, g_{1}, g_{2}, g_{3}, g_{1}^{u}, g_{1}^{v}, g_{2}^{u} g_{3}^{v}\right) \\
& =\hat{H}^{\prime}\left(A, B, C, g_{1}, g_{2}, g_{3}, g_{1}^{u}, g_{1}^{v}, g_{2}^{u} g_{3}^{v}\right)=h^{\prime}
\end{aligned}
$$

is $1 / q$. Since $q>5$, we have in particular that the probability that $h \neq h^{\prime}$ is $1-1 / q>4 / 5$.

Summing up, $\mathcal{B}$ would obtain with probability at least $\varepsilon / 2 \cdot \varepsilon / 2 \cdot(q-1 / q)>\varepsilon^{2} / 5$ two valid proofs of knowledge $\left(h, w_{1}, w_{2}\right)$ corresponding to the randomness $(x, y)$ and $\left(h^{\prime}, w_{1}^{\prime}, w_{2}^{\prime}\right)$ corresponding to the randomness $\left(x, y^{\prime}\right)$ such that $h \neq h^{\prime}$.

Since the randomness $x$ is equal until the crucial query to the random oracle $\hat{H}$, we get

- $g_{1}^{w_{1}} A^{h}=g_{1}^{w_{1}^{\prime}} A^{h^{\prime}}$ and

- $g_{1}^{w_{2}} B^{h}=g_{1}^{w_{2}^{\prime}} B^{h^{\prime}}$.

The first statement implies that

$$
w_{1}+\alpha h=w_{1}^{\prime}+\alpha h^{\prime} \bmod q,
$$

whereas the second statement implies

$$
w_{2}+\beta h=w_{2}^{\prime}+\beta h^{\prime} \bmod q .
$$

Therefore, algorithm $\mathcal{B}$ can compute

$$
\alpha=\frac{w_{1}-w_{1}^{\prime}}{h^{\prime}-h} \bmod q \quad \text { and } \quad \beta=\frac{w_{2}-w_{2}^{\prime}}{h^{\prime}-h} \bmod q .
$$

Zero knowledge. We show that a verifier does not obtain any new information from the execution of the protocol other than the proof itself. In order to prove this, we show that the verifier himself could have computed a valid proof of knowledge which is indistinguishable from a real one computed by an honest prover in the random oracle model.

In effect, the verifier can choose at random $w_{1}, w_{2}, h \in \mathbb{Z}_{q}$. Then, he can impose that

$$
\hat{H}\left(A, B, C, g_{1}, g_{2}, g_{3}, g_{1}^{w_{1}} A^{h}, g_{1}^{w_{2}} B^{h}, g_{2}^{w_{1}} g_{3}^{w_{2}} C^{h}\right)=h .
$$

Since the hash function $\hat{H}$ is assumed to behave as a totally random function (the proof is valid in the random oracle model), then this step is consistent and produces a valid proof of knowledge which is indistinguishable from a real one computed by an honest prover who knows $\alpha$ and $\beta$. This is true if the value imposed on the hash function $\hat{H}$ in the simulation does not produce a collision with previously assigned values.

\subsection{Security Analysis}

In this section, we prove that the scheme of Daza et al., explained in Section 4.1, is secure with respect to the formal model explained in Section 2.1.2 as long as the DDH problem (see Definition 2) is hard to solve. As happens with the scheme of Naor et al. [24], the proof is valid in the random oracle model for the hash functions $H$ and $\hat{H}$. In Section 5, we will explain how it is possible to erase this assumption for the hash function $H$ (this modification is valid for both schemes). This will be at the cost of a loss in efficiency. 
Theorem 1. Assume that the distributed key distribution scheme of Daza et al. can be $\left(\mathcal{U}, \mathcal{S}, \Gamma, \mathcal{A}, T, \varepsilon, Q_{\text {conf }}\right)$-broken with $Q_{H}$ queries to the random oracle $H$ and $Q_{\hat{H}}$ queries to the random oracle $\hat{H}$, satisfying $Q_{\text {conf }}<q /(2 n)$ and $Q_{\hat{H}}<q^{2} / 2$.

Then, the DDH problem can be $\left(\varepsilon^{\prime}, T^{\prime}\right)$-solved, where $\varepsilon^{\prime} \geq$ $\varepsilon / 4$ and $T^{\prime} \leq T+T_{f} Q_{H}+\left(7+n^{2}\right) T_{f} Q_{\text {conf }}$, where $T_{f}$ is the time to perform a modular exponentiation.

Proof. Let $\mathcal{D}=\left(g^{a}, g^{b}, g^{c}\right)$ be an instance of the $\mathrm{DDH}$ problem; the goal is to decide if $\mathcal{D}=\mathcal{D}_{D H}$ or $\mathcal{D}=\mathcal{D}_{\text {rand }}$. To do it, we will run the hypothetical adversary $\mathcal{F}$ against the scheme of Daza et al. We initialize the scheme with the same public parameters that appear in the given instance of the DDH problem: the primes $p$ and $q$ and the element $g$. We send to $\mathcal{F}$ the information about the sets and the structures: $\mathcal{S}, \Gamma, \mathcal{A}, \mathcal{U}$, and $\mathcal{C}$.

Since we are assuming that the hash function $H$ : $\{0,1\}^{*} \rightarrow \mathbb{G}$ behaves as a random function, we maintain a table $T A B_{H}$ as follows: When $\mathcal{F}$ asks for the value $H(C)$ for some conference $C \subset \mathcal{U}$, we look for the input $C$ in the table $T A B_{H}$. If it is already in the table, we return to $\mathcal{F}$ the corresponding output. If not, we choose at random $\rho_{C} \in \mathbb{Z}_{q}$ and define $h_{C}=H(C)=\left(g^{a}\right)^{\rho_{C}}$. We return this value to $\mathcal{F}$ and store the new relation in $T A B_{H}$. The condition $h_{C_{1}} \neq h_{C_{2}}$ must be satisfied for any two different conferences $C_{1} \neq C_{2}$.

In an analogous way, we manage a table $T A B_{\hat{H}}$ for the values of the random hash function $\hat{H}$ that is used in the proofs of knowledge.

The adversary $\mathcal{F}$ plays game $\mathcal{G}$ against the scheme and we must simulate all of the information that $\mathcal{F}$ obtains in the different steps of the game. Note that the simulation that we propose below will be consistent only in the case where $\mathcal{D}=\mathcal{D}_{D H}$ :

1. $\mathcal{F}$ chooses a subset $B \in \mathcal{A}$ of corrupted servers. Without loss of generality (adding, if necessary, some players to the original subset $B$ ), we can assume that $B$ is a maximal nonauthorized subset, that is, for any server $S_{i} \notin B$, it is fulfilled that $B \cup\left\{S_{i}\right\} \in \Gamma$.

2. The initialization phase of the scheme must be executed, which involves only the servers. The adversary obtains the public information and the private information of the corrupted servers. We proceed as follows: We compute $\operatorname{Sim}\left(B, g^{b}\right)$, which is all the information that the adversary would obtain in an execution of the joint protocol for the generation of a shared secret value $\alpha$ such that $g^{\alpha}=g^{b}$ (described in Section 3.2.1). This information, $\operatorname{Sim}\left(B, g^{b}\right)$, consists (basically) of the values $\left\{\alpha_{j}^{\prime}\right\}_{S_{j} \in B}$ and $\left\{g^{\alpha_{i}^{\prime}}\right\}_{S_{i} \in \mathcal{P}}$.

3. In Step 3 of the game, $\mathcal{F}$ chooses different conferences $C^{\prime} \subset \mathcal{U}$. For each user $U \in C^{\prime}$, with ElGamal public key $y$, the adversary $\mathcal{F}$ must receive all the information that $U$ and servers in $B$ would see in an execution of the protocol where $U$ obtains an encryption of the conference key $\kappa_{C^{\prime}}=h_{C^{\prime}}^{\alpha}=g^{a \rho_{C^{\prime}} b}$. We allow the adversary $\mathcal{F}$ to know the secret key of user $U$ and, thus, to obtain the final conference key $\kappa_{C^{\prime}}$.
We are implicitly assuming that $g^{c}=g^{a b}$. So, first, we compute an ElGamal encryption (under $U^{\prime}$ s public key, $y$ ) of the assumed final key $\kappa_{C^{\prime}}=g^{c \rho_{C^{\prime}}}$ : We choose at random $\beta \in \mathbb{Z}_{q}^{*}$ and compute

$$
r=g^{\beta} \quad \text { and } \quad s=\left(g^{c}\right)^{\rho_{C^{\prime}}} y^{\beta} .
$$

Then, we compute the partial ElGamal encryptions, starting with servers $S_{j} \in B$; for them, we know the shares $\alpha_{j}^{\prime}$, so we choose at random $\beta_{j} \in$ $\mathbb{Z}_{q}^{*}$ and compute

$$
r_{j}=g^{\beta_{j}} \quad \text { and } \quad s_{j}=\left(g^{a \rho_{C^{\prime}}}\right)^{\alpha_{j}^{\prime}} y^{\beta_{j}} .
$$

We can easily compute $\operatorname{Proof} f_{j}$ for these servers $S_{j}$ as well since we know the values $\alpha_{j}^{\prime}$ and $\beta_{j}$.

Finally, for servers $S_{i} \notin B$, we know that $B \cup\left\{S_{i}\right\} \in \Gamma$. This implies the existence of values $\lambda_{0}$ and $\left\{\lambda_{j}\right\}_{S_{j} \in B}$ such that $\psi\left(S_{i}\right)=\lambda_{0} \psi(D)+\sum_{S_{j} \in B} \lambda_{j} \psi\left(S_{j}\right)$. Then, we can compute the values

$$
r_{i}=r^{\lambda_{0}} \prod_{S_{j} \in B} r_{j}^{\lambda_{j}} \quad \text { and } \quad s_{i}=s^{\lambda_{0}} \prod_{S_{j} \in B} s_{j}^{\lambda_{j}},
$$

which form a valid ElGamal encryption of the value $h_{C^{\prime}}^{\alpha^{\prime}}=\left(g^{a \rho_{C^{\prime}}}\right)^{\alpha_{i}^{\prime}}$ under public key $y$.

To simulate Proof for servers $S_{i} \notin B$, we proceed as we described in Section 4.2. We choose at random $w_{1}, w_{2}, h \in \mathbb{Z}_{q}$ and define

$\hat{H}\left(D_{i}, r_{i}, s_{i}, g, h_{C^{\prime}}, y, g^{w_{1}} D_{i}^{h}, g^{w_{2}} r_{i}^{h}, h_{C^{\prime}}^{w_{1}} y^{w_{2}} s_{i}^{h}\right)=h$.

This relation is stored in the table $T A B_{\hat{H}}$. Note that there cannot be collisions among different simulations of proofs of knowledge because there is only one simulation for the values $D_{i}, h_{C^{\prime}}, y$ corresponding to server $S_{i}$, conference $C^{\prime}$, and user $U$ with public key $y$. However, there can be other kind of collisions if some input of $\hat{H}$ obtained in a simulation has been already asked to the random oracle by $\mathcal{F}$. If we detect such a collision, we abort the game and output a random answer to the given instance of the $\mathrm{DDH}$ problem. Since we simulate at most $n Q_{\text {conf }}$ proofs of knowledge and $\mathcal{F}$ is allowed to make at most $Q_{\hat{H}}$ queries to the random oracle $\hat{H}$, the probability $\mu$ that such a collision occurs is

$$
\mu \leq \frac{n Q_{\text {conf }} Q_{\hat{H}}}{q^{3}} .
$$

Since $Q_{\text {conf }}<q /(2 n)$ and $Q_{\hat{H}}<q^{2} / 2$, the probability that no collision occurs is $1-\mu>1 / 2$.

4. The adversary $\mathcal{F}$ chooses a conference $C$ different from the previous conferences $C^{\prime}$. We can simulate the information that $\mathcal{F}$ would obtain from the execution of the protocol for $C$, exactly in the same way as in the previous step. The difference is that now we do not allow $\mathcal{F}$ to obtain the ElGamal decryption, which is, supposedly, the final conference key $\kappa_{C}$. 
Note that this Step 4 can eventually occur in parallel to the previous Step 3; the simulation can be done in exactly the same way. As well, new key conference queries, such as those in Step 3, could be made by the adversary now for conferences $C^{\prime}$ satisfying $C^{\prime} \neq C$. They are answered exactly as explained in Step 3.

5. In Step 5, we send to $\mathcal{F}$ the value $\left(g^{c}\right)^{\rho_{C}}$. Note that, if $g^{c}=g^{a b}$, this value is exactly $\kappa_{C}$.

6. The adversary $\mathcal{F}$ outputs a bit $b^{\prime}$.

We answer to the given instance $\mathcal{D}$ of the $\mathrm{DDH}$ problem as follows: If $\mathcal{F}$ outputs 1 , we state that $\mathcal{D}$ follows the Diffie-Hellman distribution or, in other words, that $c=a b \bmod q$. If $\mathcal{F}$ outputs 0 , we state that $c \neq a b \bmod q$.

Let us compute the probability $\varepsilon^{\prime}$ that we solve the $\mathrm{DDH}$ problem correctly. If some collision happens in the management of the table $T A B_{\hat{H}}$, then we succeed with probability $1 / 2$. If no collisions occur, then we can distinguish two cases. On the one hand, if $\mathcal{D}=\mathcal{D}_{D H}$, which happens with probability $1 / 2$ since $\mathcal{D}$ is taken at random between $\mathcal{D}_{D H}$ and $\mathcal{D}_{\text {rand }}$, all of the values that $\mathcal{F}$ receives during the game are consistent and, in Step 6, he receives the correct value of $\kappa_{C}$ (that is, $b^{*}=1$ ). In this case, by hypothesis, $\mathcal{F}$ will output the correct answer $b^{\prime}=b^{*}=1$ with probability at least $\varepsilon+1 / 2$. And, with this probability, we will answer correctly that $\mathcal{D}$ follows the DiffieHellman distribution. On the other hand, if $\mathcal{D}=\mathcal{D}_{\text {rand }}$ (which also happens with probability $1 / 2$ ), all of the information that $\mathcal{F}$ receives during the game is completely independent of the bit $b^{*}$ which defines the game, so $\mathcal{F}$ will output the correct $b^{\prime}=0$ with probability $1 / 2$.

Summing up, considering all of the possible cases, we obtain that the probability of correctly solving the instance of the DDH problem is at least

$$
\mu \cdot \frac{1}{2}+(1-\mu) \cdot\left(\frac{1}{2} \cdot \frac{2 \varepsilon+1}{2}+\frac{1}{2} \cdot \frac{1}{2}\right)=\frac{1}{2}+\frac{\varepsilon(1-\mu)}{2}>\frac{1}{2}+\frac{\varepsilon}{4} .
$$

Therefore, we get $\varepsilon^{\prime} \geq \varepsilon / 4$. With respect to the execution time $T^{\prime}$ of our algorithm for solving the $\mathrm{DDH}$ problem, if we consider as significant only the time $T_{f}$ to perform modular exponentiations, then $T^{\prime}$ is the same as the execution time $T$ of $\mathcal{F}$ plus the time of answering the $Q_{H}$ queries to the hash (random) oracle $H$ plus the time of simulating the information that $\mathcal{F}$ obtains in game $\mathcal{G}$ (ElGamal partial encryptions, proofs of knowledge, etc.). Summing up, we have $T^{\prime} \leq T+T_{f} Q_{H}+\left(7+n^{2}\right) T_{f} Q_{\text {conf } f}$.

\section{A Modification to Avoid the Random ORACLE MODEL}

The security of the distributed key distribution schemes in [11], [24] can be proven in the random oracle model. This is a usual strategy in cryptography, although the assumption of the total randomness of a hash function is unrealistic. Even if a proof in the random oracle model is always better than nothing, efforts are constantly being made by cryptographers to provide protocols whose security does not rely on this assumption.
In their work, Naor et al. [24] briefly mention a possible modification in the way in which the values $h_{C}$ are computed in order to avoid the use of hash function $H$ and, therefore, the assumption of the random oracle model for this function in the security proof. We detail this modification here, which is also valid for the scheme of Daza et al. that we study in this paper. However, note that the random oracle model will still be necessary for the function $\hat{H}$ if we consider the zero-knowledge proofs of knowledge to achieve robustness against malicious adversaries (in both schemes).

The idea is that servers will jointly compute a value $h_{C} \in$ $\mathbb{G}=\langle g\rangle$ for each conference $C$ and store this relation in a table. In some sense, the management of this table by the servers plays the role of a random oracle model. Since the values $h_{C}$ can be random, servers can compute them offline in the initialization phase. Later, when a new conference key is requested, they publicly assign to this conference $C$ one of the precomputed values $h_{C}$ and store the relation in the table. When all of the users in conference $C$ have obtained the key $\kappa_{C}$, they erase this relation from the table.

Note that this solution may be suitable only for scenarios where the expected number of conferences is not too big since the size of the table that must be managed by the servers is linear on the number of expected conferences.

There are different possibilities for this joint generation of the value $h_{C}$. A first (naive) solution consists of each server $S_{i} \in \mathcal{S}$ generating, at random, a value $\mu_{C, i} \in \mathbb{Z}_{q}$ and then broadcasting the value $h_{C, i}=g^{\mu_{C, i}}$. The final value to be stored in the table is

$$
h_{C}=\prod_{S_{i} \in \mathcal{S}} h_{C, i}=g^{\sum_{S_{i} \in \mathcal{S}} \mu_{C, i}} .
$$

However, this solution requires simultaneous broadcast channels [21] for all of the servers in $\mathcal{S}$ to ensure that all of the values $h_{C, i}$ are broadcast at the same time, with independence. If not, a server $S_{j}$ corrupted by the adversary could wait and choose its value $h_{C, j}$ in order to result in a desired $h_{C}$. Later, he can do the same for a different conference $C^{\prime}$ and produce, for example, $h_{C^{\prime}}=h_{C}^{2}$. In this case, if the adversary could have access to the key $\kappa_{C}=h_{C}^{\alpha}$, then he would be able to compute the conference key $\kappa_{C^{\prime}}=h_{C^{\prime}}^{\alpha}=\kappa_{C^{\prime}}^{2}$, thus breaking the security of the scheme.

Such an assumption about simultaneous broadcast channels may be very strong in some scenarios. For this reason, an alternative (and less efficient) solution is that servers run, for each value $h_{C}$ to be computed, the protocol described in Section 3.2.1. With this last modification, the security of the two distributed key distribution schemes [11], [24] can be proven in a similar way. Since the protocol in Section 3.2.1 is simulatable, the information that the adversary obtains in the generation of the values $h_{C}$ can be perfectly simulated in the security proof. The relation between the times $T^{\prime}$ and $T$, in Theorem 1, will now include the time of simulating $Q_{\text {conf }}$ times the execution of the aforementioned protocol. Note that this use of verifiable secret sharing techniques (i.e., protocol in Section 3.2.1) to achieve "mutually independent channels" is not new; see the original paper [9] on verifiable secret sharing. 


\section{Conclusions}

In this work, we have analyzed an existing distributed key distribution scheme [11] whose security had not been studied at all. This scheme is especially suitable in real scenarios where users have low computational resources and servers are even paid in some way for doing most of the work. We have considered a formal security model for this kind of scheme. We have proven that this specific scheme achieves the desired level of security in the random oracle model as long as the DDH problem is hard to solve. To do so, we have first replaced the protocol for the joint generation of a random value in [11] with a more secure one and we have also analyzed the security of a zeroknowledge protocol, which provides robustness to the scheme.

We also discuss a modification of the scheme in [11] which is also valid for the scheme in [24], which replaces the use of a hash function with a table that must be managed by the servers. On the one hand, this results in less efficient distributed key distribution schemes, maybe only suitable for scenarios where the expected number of conferences is small, but, on the other hand, this modification allows us to avoid the strong assumption that this hash function behaves as a random oracle.

\section{ACKNOWLEDGMENTS}

The authors would like to thank the anonymous referees of the IEEE Transactions on Computers for their helpful comments about this work. The work of the first two authors was partially supported by the Spanish Ministry of Education and Science under Projects TSI2007-65406-C0302 ("eAEGIS") and CONSOLIDER CSD2007-00004 ("ARES"). The work of V. Daza was also supported by the Government of Catalonia under Grant 2005-SGR-00446. The work of J. Herranz was also supported by the Government of Catalonia under Grant 2005-SGR-00093. Finally, the work of G. Sáez was partially supported by the Spanish Ministry of Education and Science under Project TSI2006-02731.

\section{REFERENCES}

[1] M. Bellare, D. Pointcheval, and P. Rogaway, "Authenticated Key Exchange Secure against Dictionary Attacks," Proc. Int'l Conf. Theory and Application of Cryptographic Techniques, pp. 139-155, 2000.

[2] M. Bellare and P. Rogaway, "Random Oracles Are Practical: A Paradigm for Designing Efficient Protocols," Proc. First ACM Conf. Computer and Comm. Security, pp. 62-73, 1993.

[3] G.R. Blakley, "Safeguarding Cryptographic Keys," Proc. Nat'l Computer Conf., Am. Federation of Information, Processing Societies, pp. 313-317, 1979.

[4] C. Blundo, P. D'Arco, V. Daza, and C. Padró, "Bounds and Constructions for Unconditionally Secure Distributed Key Distribution Schemes for General Access Structures," Theoretical Computer Science, vol. 320, pp. 269-291, 2004.

[5] E. Bresson and D. Catalano, "Constant Round Authenticated Group Key Agreement via Distributed Computation," Proc. Seventh Int'l Workshop Practice and Theory in Public Key Cryptography, vol. 2947, pp. 115-129, 2004.

[6] E.F. Brickell, "Some Ideal Secret Sharing Schemes," J. Combinatorial Math. and Combinatorial Computing, vol. 9, pp. 105-113, 1989.
[7] M. Burmester and Y.G. Desmedt, "A Secure and Efficient Conference Key Distribution System," Proc. Int'l Conf. Theory and Application of Cryptographic Techniques, vol. 950, pp. 275-286, 1994.

[8] J. Camenisch, "Group Signature Schemes and Payment Systems Based on the Discrete Logarithm Problem," PhD thesis, ETH Zurich, Diss. ETH No. 12520, 1998.

[9] B. Chor, S. Goldwasser, S. Micali, and B. Awerbuch, "Verifiable Secret Sharing and Achieving Simultaneity in the Presence of Faults," Proc. 26th IEEE Symp. Foundations of Computer Science, pp. 383-395, 1985.

[10] P. D'Arco and D.R. Stinson, "On Unconditionally Secure Robust Distributed Key Distribution Centers," Proc. Eighth Int'l Conf. Theory and Application of Cryptology and Information Security, pp. 346-363, 2002.

[11] V. Daza, J. Herranz, C. Padró, and G. Sáez, “A Distributed and Computationally Secure Key Distribution Scheme," Proc. Fifth Information Security Conf., pp. 342-356, 2002.

[12] V. Daza, J. Herranz, and G. Sáez, "Constructing General Dynamic Group Key Distribution Schemes with Decentralized User Join," Proc. Eighth Australasian Conf. Information Security and Privacy, pp. 464-475, 2003.

[13] V. Daza, J. Herranz, and G. Sáez, "Protocols Useful on the Internet from Distributed Signature Schemes," Int'l J. Information Security, vol. 3, no. 2, pp. 61-69, 2004.

[14] W. Diffie and M.E. Hellman, "New Directions in Cryptography," IEEE Trans. Information Theory, vol. 22, no. 6, pp. 644-654, 1976.

[15] P. Feldman, "A Practical Scheme for Non-Interactive Verifiable Secret Sharing," Proc. 28th IEEE Symp. Foundations of Computer Science, pp. 427-437, 1987.

[16] A. Fiat and A. Shamir, "How to Prove Yourself: Practical Solutions of Identification and Signature Problems," Proc. Advances in Cryptology, pp. 186-194, 1986.

[17] T. ElGamal, "A Public Key Cryptosystem and a Signature Scheme Based on Discrete Logarithms," IEEE Trans. Information Theory, vol. 31, pp. 469-472, 1985.

[18] R. Gennaro, S. Jarecki, H. Krawczyk, and T. Rabin, "Robust Threshold DSS Signatures," Proc. Int'l Conf. Theory and Application of Cryptographic Techniques, pp. 354-371, 1996.

[19] R. Gennaro, S. Jarecki, H. Krawczyk, and T. Rabin, "Secure Distributed Key Generation for Discrete-Log Based Cryptosystems," Proc. Int'l Conf. Theory and Application of Cryptographic Techniques, vol. 1592, pp. 295-310, 1999.

[20] S. Goldwasser and S. Micali, "Probabilistic Encryption," J. Computer and System Sciences, vol. 28, pp. 270-299, 1984.

[21] A. Hevia and D. Micciancio, "Simultaneous Broadcast Revisited," Proc. 24th Ann. ACM Symp. Principles of Distributed Computing, pp. 324-333, 2005.

[22] J. Katz and M. Yung, "Scalable Protocols for Authenticated Group Key Exchange," Proc. Advances in Cryptology, pp. 110-125, 2003.

[23] H. Kurnio, R. Safavi-Naini, and H. Wang, "A Group Key Distribution Scheme with Decentralised User Join," Proc. Third Conf. Security in Comm. Networks, pp. 146-163, 2002.

[24] M. Naor, B. Pinkas, and O. Reingold, "Distributed PseudoRandom Functions and KDCs," Proc. Int'l Conf. Theory and Application of Cryptographic Techniques, pp. 327-346, 1999.

[25] R.M. Needham and M.D. Schroeder, "Using Encryption for Authentication in Large Networks of Computers," Comm. ACM, vol. 21, pp. 993-999, 1978.

[26] T.P. Pedersen, "Non-Interactive and Information-Theoretic Secure Verifiable Secret Sharing," Proc. Advances in Cryptology, pp. 129140, 1991.

[27] D. Pointcheval and J. Stern, "Security Arguments for Digital Signatures and Blind Signatures," J. Cryptology, vol. 13, no. 3, pp. 361-396, 2000.

[28] C.P. Schnorr, "Efficient Signature Generation by Smart Cards," J. Cryptology, vol. 4, pp. 161-174, 1991.

[29] A. Shamir, "How to Share a Secret," Comm. ACM, vol. 22, pp. 612$613,1979$.

[30] G.J. Simmons, W. Jackson, and K. Martin, "The Geometry of Secret Sharing Schemes," Bull. ICA, vol. 1, pp. 71-88, 1991. 


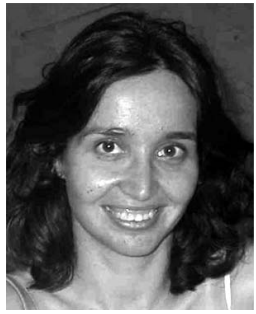

Vanesa Daza received the degree in mathematics from the University of Barcelona (UB), Spain, in 1999 and the PhD degree in mathematics from the Technical University of Catalonia (UPC), Barcelona, in 2004. Afterward, she joined a crypto-based security company as a senior researcher. She currently holds a lecturer position in the CRISES research group in the Rovira i Virgili University (URV), Tarragona, Spain. Her research interests are mainly related to distributed cryptography, including secret sharing and multiparty computation.

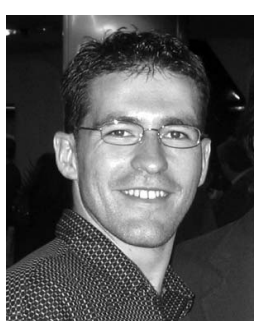

Javier Herranz received the degree in mathematics and the PhD degree in applied mathematics from the Technical University of Catalonia (UPC), Barcelona, in 2000 and 2005, respectively. After that, he spent nine months at the École Polytechnique, Palaiseau, France, and nine months at the Centrum voor Wiskunde en Informatica (CWI), The Netherlands, as a postdoctoral researcher, granted with an ERCIM fellowship (from May 2005 to October 2006). He is currently a postdoctoral researcher at IIIA-CSIC, Bellaterra, Spain. His research interests are mostly related to cryptography, especially to digital signatures and distributed cryptographic protocols.

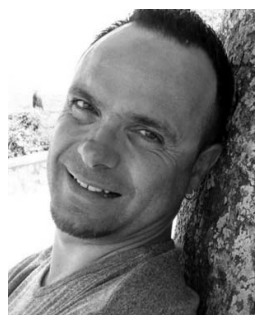

Germán Sáez received the degree in mathematics from the University of Barcelona (UB), Spain, in 1987 and the PhD degree in mathematics from the Technical University of Catalonia (UPC), Barcelona, in 1998. He is an associate professor in the Department of Applied Mathematics IV at UPC. He was the general cochair of Eurocrypt '07. He belongs to the Research Group on Mathematics Applied to Cryptography (MAK) at UPC. His research interests include secret sharing schemes and digital signatures in distributed scenarios.

$\triangleright$ For more information on this or any other computing topic, please visit our Digital Library at www.computer.org/publications/dlib. 